\title{
The -172A>G Polymorphism in ADAM17 Promoter Region Enhances Its Binding to EGR1 and Confers Susceptibility to Sepsis Progression
}

\section{Yiming Shao}

The Intensive Care Unit, The Second Affiliated Hospital of Guangdong Medical University

Wenying Zhang

The Intensive Care Unit, The Second Affiliated Hospital of Guangdong Medical University

Tian Zhao

The Intensive Care Unit, The Second Affiliated Hospital of Guangdong Medical University

\section{Furong Lu}

The Intensive Care Unit, The Second Affiliated Hospital of Guangdong Medical University

\section{Yuan Hong}

The Intensive Care Unit, The Second Affiliated Hospital of Guangdong Medical University

\section{Lizhen Liu}

The Intensive Care Unit, The Second Affiliated Hospital of Guangdong Medical University

\section{Yujie Cai}

Institue of Neurology, Guangdong Key Laboratory of Age-Related Cardiac and Cerebral Diseases, Affiliated Hospital of Guangdong Medical University

\section{Ning Wei}

The Intensive Care Unit, The Second Affiliated Hospital of Guangdong Medical University

\section{HongXiao Cheng}

The Intensive Care Unit, The Second Affiliated Hospital of Guangdong Medical University

\section{Feng Chen}

Institute of Neurology, Guangdong Key Laboratory of Age-Related Cardiac and Cerebral Diseases, Affiliated Hospital of Guangdong Medical University

\section{Junbing He}

The Intensive Care Unit, Jieyang Affiliated Hospital, SunYat-sen University

Lili Cui ( $\square$ cuilili@gdmu.edu.cn )

Institute of Neurology, Guangdong Key Laboratory of Age-Related Cardiac and Cerebral Diseases, Affiliated Hospital of Guangdong Medical University, Renmin street south 57, Xiashan district, Zhanjiang City 524001, Guangdong Province, PR China https://orcid.org/0000-0003-0273-2531

\section{Research}

Keywords: ADAM17, EGR1, Polymorphism, Sepsis, inflammation

Posted Date: September 9th, 2020

DOI: https://doi.org/10.21203/rs.3.rs-73158/v1

License: @ (7) This work is licensed under a Creative Commons Attribution 4.0 International License. Read Full License 


\section{Abstract}

Background: A disintegrin and metalloproteinase 17 (ADAM17) is a proteolytic cleaving protein with a crucial function in inflammatory responses, especially sepsis. But the clear role of ADAM17 in sepsis and the underlying mechanism remained unknown. In this study, we aim to determine the clinical relevance of the ADAM17 rs12692386 (-172A>G) promoter polymorphism in sepsis progression and to further explore the effect and mechanism of the early growth response 1 (EGR1) /ADAM17 pathway in the inflammatory process following sepsis.

Methods: A case-control study with a total of 903 sepsis patients and 1150 controls were enrolled to determine the association of ADAM17 -172A>G polymorphism with sepsis. The transcription factor binding to the promoter region of ADAM17 gene was predicted by bioinformatics analysis and verified by Chromatin Immunoprecipitation (ChIP) and luciferase assays. Quantitative real-time PCR and Western blot were performed to detect EGR1 and ADAM17 expression. Cytokine production was detected by enzyme-linked immunosorbent assay. The effect of EGR1/ADAM17 pathway on sepsis-induced inflammatory responses was evaluated in EGR1silenced cells and endotoxemia mouse model.

Results: Patients with sepsis subtype exhibited significantly lower frequencies of the -172AG/GG genotypes compared to those with severe sepsis ( $29.3 \%$ vs. $45.9 \%, P=0.0002)$ or septic shock ( $29.3 \%$ vs. $42.4 \%, P=0.0032)$. The frequency of $-172 \mathrm{G}$ allele in the 28 -day non-surviving patients was significantly higher than that in the surviving patients $(29.3 \%$ vs. $21.8 \%, P=0.0188)$. The results of in vitro lipopolysaccharide-stimulated and luciferase assays indicated that the -172 A-to-G variation could functionally upregulate promoter activity and transcription of ADAM17 gene via enhancing the binding affinity of its promoter region with the EGR1. The ChIP assay identified the direct interaction. Further studies demonstrated that the EGR1/ADAM17 intervention could significantly decrease the pro-inflammatory cytokine secretion in vitro and improve the survival and inflammatory response of sepsis mouse model.

Conclusions: These results provide evidence that the ADAM17 -172A $>$ G polymorphism can functionally enhance ADAM17 expression and promote sepsis-induced inflammatory responses via the EGR1/ADAM17 pathway, which ultimately promote sepsis progression and poor prognosis.

\section{Introduction}

Sepsis is a complex disorder resulting from the systemic dysregulation of host responses to infection, which can result in the development of acute organ dysfunction with a high risk of death [1,2]. Despite advancements in treatment strategies, there is currently no approved specific and effective treatment for this disorder [3]. Recent epidemiological studies have demonstrated that genetic factors play a crucial role in the susceptibility to and progression of sepsis [4]. Diagnostic strategies based on targeted gene sequencing and therapies directed against genetic polymorphisms might improve the prognosis of sepsis patients.

A disintegrin and metalloproteinase 17 (ADAM17), a member of the ADAM family, is involved in the shedding of more than 80 cellular substrates, including tumor necrosis factor-alpha (TNF-a), interleukin-6 receptor (IL-6R) and vascular cell adhesion molecule-1 (VCAM-1), and the initiation of several inflammatory signaling pathways that are hypothesized to play critical roles in sepsis [5-10]. Several lines of evidence indicate that ADAM17 is highly expressed in sepsis patients and animal models of sepsis, which is markedly associated with organ dysfunction and mortality [11-13]. The inhibition or genomic deletion of ADAM17 contributes to bacterial clearance and a decreased inflammatory response and offers mice a survival benefit following sepsis [14-16], and these findings indicate an important role of ADAM17 in the pathogenesis of sepsis. The human ADAM17 gene is located on chromosome 2 and contains 19 exons. Accumulating evidence demonstrates that single-nucleotide polymorphisms (SNPs) in the ADAM17 gene are associated with risk for various inflammation-related diseases, such as ischemic stroke, Kawasaki disease and cardiovascular death [17-19]. Our previous study revealed, for the first time that ADAM17 -172A $>$ G may act as a functional SNP involved in sepsis progression [13]. However, the mechanism through which this SNP affects ADAM17 expression and sepsis progression remains unknown, and a larger and independent validation cohort study is needed to verify our results.

EGR1 is a transcription factor involved in the regulation of diverse cellular functions by regulating the transcription of a wide array of downstream genes $[20,21]$. This transcription factor can be upregulated by cytokines, hypoxia, vascular injury and septic shock [22-25]. In an LPS-induced endotoxemia mouse model, EGR-1 contributes to the sustainable appearance of inflammatory mediators in the kidneys and lungs [26], and EGR1 could be induced to rise significantly in sepsis within 30 minutes [27]. Additionally, EGR1 is involved in the modulation of the ERK, JNK and p38 MAPK pathways and the production of inflammatory cytokines [28, 29]. A 
bioinformatics analysis revealed a conserved putative transcription factor-binding site for EGR1 in the 5'-untranslated region (5'-UTR) of human ADAM17. However, the specific role of the -172A>G polymorphism and EGR1 in the regulation of ADAM17 transcriptional expression and sepsis remains unclear. Thus, we expanded the previous studied samples and enrolled two additional populations to validate the clinical association of the $-172 A>G$ polymorphism with sepsis. Furthermore, functional in vitro and vivo assays were conducted to characterize the role of $-172 A>G$ and EGR1 in the modulation of inflammatory responses in sepsis by regulating ADAM17 gene transcription. The mechanistic characterization of this sepsis-associated functional polymorphism might provide new opportunities for the development of targeted treatments for sepsis.

\section{Methods}

Study population This study was approved by the Ethics Committees of the participating hospitals, and written informed consent was signed by all enrolled subjects or their families prior to enrollment. The studied subjects were recruited from three hospitals in North, Central and South China: Affiliated Hospital of Guangdong Medical University, Zhanjiang, Guangdong Province; Central Hospital of Wuhan, Wuhan, Hubei Province; and Second Affiliated Hospital of Harbin Medical University, Harbin, Heilongjiang Province. The patients were enrolled in the study if they met the following inclusion criteria: 1) the patients were older than 18 years of age and belonged to the Chinese Han population; 2) the diagnosis of sepsis, severe sepsis, or septic shock was established according to the International Sepsis Definitions Conference [30]; and 3) the patients had a probability of survival greater than 24 hours. Patients were excluded from this study if they had diabetes, malignant tumors, human immunodeficiency virus, or autoimmune diseases or were pregnant, readmitted to a hospital or receiving immunosuppressive therapy, corticosteroid therapy or chemoradiotherapy. The healthy controls, which were enrolled at the health examination center at each hospital, had no history of sepsis, no recent acute illness and no chronic illness, such as autoimmune diseases, diabetes, cancer and major cardiac, renal, hepatic, and endocrinological disorders. The healthy controls also belonged to the Chinese Han population, and their healthy status was determined by reviewing the medical examination reports and questioning the participants. The age, sex, source of infection, cause of infection, pathogenic bacteria, and Acute Physiology and Chronic Health Evaluation (APACHE) II results of each patient were documented, and blood samples were collected within 12 hours after the diagnosis of sepsis, severe sepsis, or septic shock was established. The survival of all the patients was observed for a 28-day period. The participants' confidentiality was preserved according to the guidelines for studies of human subjects. DNA isolation and genotyping Genomic DNA was isolated from Peripheral blood mononuclear cells (PBMCs) using the TIANamp Blood DNA Kit (TianGen Biotech, Beijing, China) according to the vendor's recommendations. The ADAM17 -172A>G polymorphism was genotyped using the ABI PRISM® SNaPshot ${ }^{\text {TM }}$ Multiplex Kit (Applied Biosystems, Carlsbad, CA, USA). The sequences of the forward and reverse primers were 5'-GGCCTAGCCCCTCAATCCTCTT-3' and 5'TTTTTTTGGTAACGCCACCTG -CCTTC-3', respectively. The obtained data were analyzed using Gene Mapper 4.1 (Applied Biosystems, Foster City, CA, USA). Mononuclear cell isolation,cell culture and LPS stimulation PBMCs were isolated from the blood samples collected from the sepsis patients and healthy individuals by density gradient centrifugation using Lymphoprep ${ }^{\text {TM }}$ (Axis-Shield PoCAS, Oslo, Norway) for the analysis of ADAM17 expression. The PBMCs from the 18 healthy individuals were randomly selected for in vitro LPS stimulation experiments, and these cells were stimulated with LPS at a dose of $800 \mathrm{ng} / \mathrm{mL}$ for 6 hours. Mouse leukemia cells of monocyte macrophage (RAW264.7 cells), human umbilical vascular endothelial cells (HUVECs) and human embryonic kidney cells (HEK293T) were obtained from American Type Culture Collection (ATCC; Manassas, VA, USA). The cells were grown at $37^{\circ} \mathrm{C}$ with $5 \%$ CO2 in DMEM or RPMI 1640 medium (Gibco, USA) supplemented with 10\% fetal bovine serum (Invitrogen, USA) and penicillin/streptomycin (Sigma-Aldrich, USA). Plasmid transfection and virus infection Plasmids for the overexpression of human pCMV-EGR1 were obtained from Longqian Biotech (Shanghai, China). Prior to transfection, the cells were seeded in six-well plates and cultured in medium without antibiotics until the cell confluency reached $50-70 \%$. Transient transfections were performed using Lipofectamine 2000 (Invitrogen) according to the manufacturer's recommended protocol. In brief, Lipofectamine 2000 and the plasmids (2-4 $\mu \mathrm{g} /$ well) were separately diluted in Opti-MEM (Invitrogen) for 5 minutes and then mixed, and the resulting mixture was incubated for 25 minutes. The Lipofectamine-DNA complexes were then added to the cell medium, and the cells were cultured for 6 hours with these complexes in the absence of fetal bovine serum or antibiotics. The culture medium was subsequently replaced with serum-containing DMEM or RPMI. The EGR1 adenovirus packages used for the overexpression or silencing experiments with RAW264.7 cells and HUVECs were designed and synthesized by Shanghai Genechem Co., Ltd. (China). Cells were infected with the resultant adenoviruses containing EGR1 (Ad-EGR1) and EGR1-RNAi (Ad-EGR1-RNAi) to establish stably overexpressing/silenced cell lines. The empty adenovirus vector Ad-Control and Ad-negative control (Ad-NC) were used as controls for Ad-EGR1 and Ad-EGR1RNAi, respectively. The expression of EGR1 in the cells 24-48 hours after infection was detected by Western blotting and quantitative real-time PCR (qRT-PCR). Animal treatments C57BL/6 mice (8-12 weeks, males and females in half) were purchased from Guangdong 
Laboratory Animal Center (Guangzhou, China). The mice used for the experiments in this study were bred and maintained under conventional housing conditions in our animal facility. The animal experimental protocols were reviewed and approved by the Ethics Committee of Affiliated Hospital of Guangdong Medical University, China. EGR1 antisense oligonucleotide (As-ODN) (5'TACCGTCGCCGGTTC-3') was constructed to inhibit EGR1 by targeting the corresponding DNA sequence. The EGR1 sense ODN (SODN) (5'-TCGTGCCGCTGCCAT-3') was used as a negative control [30,31]. All ODNs were synthesized by Takara Biomedical Technology Co., Ltd. The mice were randomly divided into three groups: Control, LPS and As. To establish the LPS-induced endotoxemia model, the mice were intraperitoneally injected with LPS (Escherichia coli 055:B5, Sigma-Aldrich, USA) at a dose of 10 $\mathrm{mg} / \mathrm{kg}$. The ODNs were delivered into the mice via an intraperitoneal injection 1 hour prior to establishment of the endotoxemia model: the mice belonging to the LPS and Control groups were administered S-ODN, whereas an equivalent volume of As-ODN was injected into the mice of the As group. Six hours after establishment of the endotoxemia model, blood was collected from the mice and centrifuged for measurement of the serum concentrations of TNF-a, IL- 6 and IL-1 $\beta$ using each specific enzyme-linked immunosorbent assay (ELISA) kits. Lung and liver tissues were collected for the detection of ADAM17 and EGR1 mRNA expression. For the survival experiments, the mice were intraperitoneally injected with LPS $(20 \mathrm{mg} / \mathrm{kg})$ and monitored for 72 hours. Total RNA isolation and quantitative real-time PCR Total RNA from the PBMCs, cells or tissues was extracted using the TRIzol reagent (Invitrogen), and complementary DNA (cDNA) was synthesized from $1 \mu \mathrm{g}$ of total pure RNA using the PrimeScriptTM RT reagent kit with cDNA Eraser (Takara) in accordance to the manufacturer's recommended protocol. The primer sequences used in this study were designed by Sangon Biotech (see Additional file 1). Two-step amplification was performed with SYBR green (Takara) using $1 \mu \mathrm{L}$ of cDNA in a 10- $\mu \mathrm{L}$ reaction system. The above reaction was performed using a LightCycler 96 sequence detector system (Roche Applied Science, Laval, Quebec, Canada) under the following reaction conditions: $95^{\circ} \mathrm{C} / 600$ seconds and 40 cycles of $95{ }^{\circ} \mathrm{C} / 15$ seconds and $52{ }^{\circ} \mathrm{C} / 60$ seconds. The $2-\triangle \Delta C T$ method was used to calculate the relative RNA expression levels of the genes of interest. Glyceraldehyde 3-phosphate dehydrogenase (GAPDH) was used as an internal control. Western blot analysis Cells or tissues were harvested at the indicated times and washed twice with ice-cold PBS. The cells were then lysed with RIPA buffer containing $2 \%$ PICT and 1\% PMSF. The protein concentrations were determined using the BCA protein assay kit (Thermo Fisher Scientific, USA). The following primary antibodies were used: anti-ADAM17 (rabbit polyclonal, diluted at 1:1000, Abcam, UK), anti-EGR1 (rabbit polyclonal, 1:1000, Abcam, UK), anti-SP1 (rabbit polyclonal, 1:1000, Abcam, UK), anti-AP2a (rabbit polyclonal, 1:500, Abcam, UK), and anti- $\beta$-actin antibody (rabbit polyclonal, 1:2000, CST, USA). Cytokine measurements The concentrations of IL-1 $\beta$, IL-6, and TNF-a in the supernatants obtained from cell culture medium or animal plasma were measured using each specific ELISA kits according to the manufacturer's instructions. The following ELISA kits were used: TNF-a, IL-6, and IL-1 $\beta$ (Boster Biological Technology, USA). Flow cytometric analysis Cell apoptosis was detected by flow cytometry using the Annexin V-PE/7-AAD Apoptosis Detection Kit (YEASEN Biotechnology, Shanghai, China) following the manufacturer's instructions. The cells were harvested, washed twice with ice-cold PBS, and resuspended to a density of $1 \times 106$ cells $/ \mathrm{mL}$ in binding buffer. The cells were mixed with $5 \mu \mathrm{L}$ of Annexin V-PE and $10 \mu \mathrm{L}$ of 7$\mathrm{AAD}$ and incubated at room temperature for 15 minutes. The cells were subsequently mixed with $400 \mu \mathrm{L}$ of binding buffer and detected by flow cytometry. Luciferase plasmid constructs and luciferase reporter assay A luciferase assay was performed to determine the role of rs12692386 A>G in the promoter activities of the ADAM17 gene. Briefly, the human ADAM17 promoter sequence carrying rs12692386 (A or $\mathrm{G}$ allele at position -172) was cloned into pGL3 luciferase reporter vectors (Promega, Madison, WI, USA). The primers F-5'-CTAGCAAAATAGGCTGTCC-3' and R-5'-GCCGGGCCTTTCTTTATG-3' were used to obtain the promoter gene fragment of ADAM17 by PCR amplification, and the pGL3-ADAM17-A and pGL3-ADAM17-G plasmids were ultimately obtained. HEK293T cells were seeded ( $2 \times 107$ cells/well, 24-well plate) and cotransfected with $4 \mu \mathrm{g}$ (per well) of the pGL3-ADAM17-A/G reporter plasmid, $4 \mu \mathrm{g}$ (per well) of the transcription factor-overexpressing plasmid and $0.01 \mu \mathrm{g}$ (per well) of the pRL-TK plasmid using Lipofectamine 2000 (Invitrogen, USA). pRL-TK was used as the internal control according to the manufacturer's instructions. Forty-eight hours after transfection, the cells were harvested and lysed, and the level of luciferase activity was assayed using the dual-luciferase reporter assay system (Promega, USA). Firefly luciferase activity was normalized to Renilla luciferase activity. Chromatin immunoprecipitation (ChIP)-qPCR ChIP assays were performed the standard protocol with some modifications. ChIP coupled with quantitative PCR was used to investigate the protein-DNA interactions at binding sites in the ADAM17 gene. Briefly, chromatin was cross-linked with transcription factors, sheared into fragments (100-750 bp) by sonication and immunoprecipitated using antibody against EGR1 (CST, USA). The recovered DNA was analyzed by PCR using primers flanking the putative transcription factor-binding sites as indicated. The primers used for PCR of the ChIP fragments were as follows: F-5'-CGTGGGCGGGGCAAG-3' and R-5'GCAGGTGGCGTTACCAA -AGG-3'. The PCR program was $95^{\circ} \mathrm{C}$ for 30 seconds followed by 40 cycles of $95^{\circ} \mathrm{C}$ for 5 seconds and $60^{\circ} \mathrm{C}$ for 30 seconds. Bioinformatics analysis In order to predict the possible transcription factors that might bind to the promoter region of the ADAM17 gene, we submitted a 200-bp sequence around the - 172 position in the ADAM17 gene promoter to the following three transcription factor prediction websites: http://tfbind.hgc.jp/, http://jaspar.genereg.net/cgi-bin/jaspar_db.pl, and http://gene 
regulation. com/pub/programs/alibaba2/index.html. The final set of transcription factors was obtained by combining the results obtained using these three software packages (see Additional file 2). Statistical analysis The association between ADAM17 polymorphism and sepsis was determined using the Chi-squared test or Fisher's exact test, and the sample sizes were based on our previous experience. The Benjamin-Hochberg procedure for multiple testing correction was used to analyze the false discovery rate, and $\mathrm{P}<0.05$ after Benjamin-Hochberg correction for multiple testing was considered significant. The survival curves were plotted using the Kaplan-Meier method and compared using the log-rank test. Analysis of clinical variables such as sex were analyzed by the Chi-squared test. Image $\mathrm{J}$ software was used for densitometry quantification of the Western blot bands. The values are shown as the mean \pm standard deviation (SD). The expression of ADAM17 and EGR1, and cytokine levels in the independent groups were compared using the non-parametric Mann-Whitney U test (two-tailed). Cost outliers were excluded from the analysis. The statistical analyses were performed using SPSS 19.0 (IBM, NY, USA) or GraphPad Prism 6.0 (GraphPad Software Inc., San Diego, CA, USA), and

$P$ value less than 0.05 was considered statistically significant. Additional details are provided in the figure legends, where appropriate.

\section{Results}

\section{Study population and clinical characteristics}

A total of 903 sepsis patients (mean age: $60.9 \pm 17.1$ years; $31.7 \%$ female) and 1,150 healthy individuals (mean age: $48.1 \pm 15.2$ years; $38.1 \%$ female) were enrolled in this study between February 2013 and June 2018. The Consolidated Standards of Reporting Trials (CONSORT) flowchart of this study is shown in Additional file 3. The clinical characteristics of the enrolled subjects (903 sepsis patients) are presented in Table 1. The 903 septic cases encompassed 181 patients with sepsis subtypes (20.0\%), 368 patients with severe sepsis (40.8\%), and 354 patients with septic shock (39.2\%). The most common source of infection was respiratory tract infection $(512 / 903,56.7 \%)$, followed by Abdominal infection $(112 / 903,12.4 \%)$. The main pathogens identified in this study were Acinetobacterbaumannii (237/903, 26.2\%), P. aeruginosa (109/903, 12.1\%), Escherichia coli (97/903, 10.7\%), Staphylococcus aureus (79/903, 8.7\%), and Klebsiella pneumoniae (61/903, 6.8\%). Gram-positive and gram-negative infections accounted for $12.1 \%$ $(109 / 903)$ and $32.8 \%(296 / 903)$ of all the infections, respectively, and patients with polymicrobial infections comprised $12.1 \%$ $(109 / 903)$ of all the patients.

\section{Effect of ADAM17 genetic variations on susceptibility to sepsis development}

A total of 903 sepsis cases were separated according to the severity of sepsis into three subgroups, namely the sepsis subtype, severe sepsis and septic shock subgroups, to evaluate the association of the ADAM17-172A>G polymorphism with sepsis progression (Table 2). Our data showed that the frequencies of the AG/GG genotypes and G allele of rs 12692386 in the sepsis subtype subgroup significantly differed from those in the severe sepsis subgroup $(29.3 \%$ vs. $45.9 \%, P=0.0005$ for genotype; $17.1 \%$ vs. $25.8 \%, P=0.0039$ for allele) and septic shock subgroup ( $29.3 \%$ vs. $42.4 \%, P=0.0032$ for genotype; $17.1 \%$ vs. $23.3 \%, P=0.0193$ for allele), which suggests a functional role for the AG/GG genotype in promoting the progression of sepsis from sepsis subtype to severe sepsis and septic shock. Additionally, our data showed no significant differences in genotype/allele frequencies of ADAM17 $-172 A>G$ polymorphism between patients and healthy controls (all $P>0.05$ ), which suggests that this SNP might not influence susceptibility to sepsis (Table 3 ).

The 28-day ICU mortality was also analyzed to evaluate the effect of this ADAM17 SNP on the clinical outcome of sepsis patients. As presented in Table 4, the frequencies of non-survivors among the sepsis patients with the $-172 \mathrm{AG} / \mathrm{GG}$ genotypes and $\mathrm{G}$ allele were distinctly higher than those among patients with the AA genotype $(50.7 \%$ vs. $39.3 \%, \mathrm{OR}=0.630,95 \% \mathrm{Cl}=0.444-0.894, P=0.0188)$ and $\mathrm{A}$ allele $(29.3 \%$ vs. $21.8 \%, \mathrm{OR}=1.482,95 \% \mathrm{Cl}=1.125-1.952, P=0.0188)$. In addition, the Kaplan-Meier survival analysis indicated that the 28-day survival in septic patients with -172AG/GG genotypes was much worse than in the AA genotype carriers (log-rank test 4.696, $P=0.030$ ) (see Additional file 4).

\section{Effect of ADAM17-172A>G polymorphism on the expression of ADAM17 and proinflammatory cytokines}

We divided 80 randomly selected sepsis cases into subgroups based on organ failure and the patients' 28-day mortality, and statistically significant differences in ADAM17 expression were observed between the multiple organ dysfunction (MODS) and nonMODS subgroups (Fig. 1a). ADAM17 expression was significantly decreased in the sepsis patients who survived during the 28-day period compared with the patients who did not survive (Fig. 1a). To confirm the impact of ADAM17 rs12692386 polymorphisms on ADAM17 expression in vitro, PBMCs were isolated from 18 randomly selected healthy individuals and stimulated with LPS (800 
$\mathrm{ng} / \mathrm{mL}$ ) for 6 hours in vitro. ADAM17 expression was significantly increased in the LPS-stimulated PBMCs compared with the control PBMCs (Fig. 1b). Additionally, we analyzed the secretion of cytokines in the cell culture medium and found significantly higher concentrations of TNF-a, IL-1 $\beta$ and IL- 6 in the culture medium from LPS-stimulated PBMCs than in that from control PBMCs. Furthermore, PBMCs with the -172AG/GG genotypes exhibited significantly higher expression levels of ADAM17 than those carrying the AA genotype upon LPS stimulation (Fig. 1c). Moreover, we detected the impact of this ADAM17 genetic variation on the expression levels of cytokines. The concentrations of TNF-a $(350.5 \pm 37.6 \mathrm{pg} / \mathrm{mL}, P=0.0009)$ and IL-6 $(958.6 \pm 84.0 \mathrm{pg} / \mathrm{ml}, P=0.013)$ in LPS-stimulated PBMCs with the AG/GG genotypes were clearly higher than those with the AA genotype $(149.9 \pm 65.5 \mathrm{pg} / \mathrm{ml}$ and $625.0 \pm 300.4 \mathrm{pg} / \mathrm{ml}$, respectively) (Fig. 1c).

\section{EGR1 expression is upregulated in sepsis and is associated with ADAM17}

Because the $-172 A>G$ polymorphism is located in the promoter region of ADAM17 gene, we speculated that the A-to-G nucleotide exchange influences the binding affinity of transcription factors to this site and thereby contributes to the modulation of ADAM17 gene expression. A bioinformatics analysis predicted that three transcription factors, namely, EGR1, specificity protein 1 (SP1) and TFAP2A (AP2a), would bind to the -172A>G promoter region in an allele-specific manner (Fig. 2a). To investigate whether these transcription factors are involved in sepsis, we detected their expression in LPS-stimulated HUVECs and RAW264.7 cells and found that the expression of EGR1, but not SP1 and AP2a, was significantly increased in LPS-pretreated cells (Fig. 2b-d). Moreover, in agreement with the trend found for ADAM17 expression in these two cell lines, LPS stimulation of RAW 264.7 cells notably increased EGR1 expression to a significant level at 0.5 hours and to a peak level at 1 hour, and the peak time was delayed in HUVECs (Fig. 2e). Importantly, EGR1 mRNA expression was obviously higher in sepsis patients than in healthy controls (Fig. 2f). We further analyzed PBMCs isolated from healthy controls and found that the LPS-stimulated PBMCs showed significantly higher expression of EGR1 than the control PBMCs (Fig. 2g).

\section{ADAM17 -172A>G polymorphism upregulates the ADAM17 gene expression via enhancing the binding affinity of its promoter region with the transcription factor EGR1}

Because the $-172 A>G$ polymorphism is located in a potential EGR1-binding sequence in the ADAM17 promoter region, ChIP-qPCR and luciferase reporter assays were performed to determine the binding of EGR1 to the -172 position in the ADAM17 promoter region and to distinguish the differences in binding capacity between the rs12692386 A and G alleles to EGR1. ChIP-qPCR conducted with HUVECs indicated that EGR1 bound to the promoter region of the ADAM17 gene (Fig. 3a,b). For the luciferase reporter assays, a 6307bp ADAM17 promoter- luciferase reporter and the corresponding reporter with a single point mutation (A-to-G) on the ADAM17 rs12692386 site were constructed, and these constructs were cotransfected into HEK293T cells with pCMV-EGR1 (Fig. 3c). As shown in Fig. 3d, EGR1-overexpressing HEK293T cells transfected with pGL3-G exhibited significantly higher luciferase activities than cells transfected with pGL3-A, which indicates that ADAM17-172A>G contributes to the increased binding affinity of EGR1 to the ADAM17 promoter. We further investigated the impact of EGR1 on the regulation of ADAM17 expression in an LPS-induced cell model of sepsis. The construction and efficiency of infection of EGR1 overexpressed and silent adenovirus were presented (see Additional file 5). EGR1-overexpressing or EGR1-silenced virus successfully infected RAW264.7 cells and HUVECs (Fig. 3e). The presence of EGR1 significantly increased ADAM17 expression in LPS-induced cells, whereas these effects were completely abrogated by EGR1 knockdown (Fig. 3f). These findings confirm a positive role of EGR1 in the modulation of ADAM17 expression in sepsis.

\section{Effects of the EGR1/ADAM17 signaling pathway on proinflammatory cytokine secretion and apoptosis in vitro}

The concentrations of TNF- $a$, IL-1 $\beta$ and IL- 6 in and the apoptosis rate of LPS-pretreated cells were then analyzed to confirm the impact of the EGR1/ADAM17 signaling pathway on sepsis. Our results showed that EGR1-silenced RAW264.7 cells displayed significantly lower expression levels of TNF-a $(31.5 \pm 3.1 \mathrm{pg} / \mathrm{mL}, P=0.029)$ and IL-6 $(144.5 \pm 33.1 \mathrm{pg} / \mathrm{mL}, P=0.029)$ compared with the control cells $(47.5 \pm 2.1 \mathrm{pg} / \mathrm{mL}$ and $417.3 \pm 1.4 \mathrm{pg} / \mathrm{mL}$, respectively) upon LPS stimulation. Decreased expression of TNF-a(17.3 \pm 1.7 $\mathrm{pg} / \mathrm{mL}, P=0.029)$ and IL-1 $\beta(4.4 \pm 2.9 \mathrm{pg} / \mathrm{mL}, P=0.029)$ was also observed in LPS-stimulated EGR1-silenced HUVECs compared with those in LPS-stimulated cells ( $30.7 \pm 4.9 \mathrm{pg} / \mathrm{mL}$ and $15.7 \pm 2.9 \mathrm{pg} / \mathrm{mL}$, respectively) (Fig. 4a). In addition, the silencing of EGR1 through EGR1-RNAi infection significantly decreased the apoptotic rate of RAW264.7 cells upon LPS stimulation (Fig. 4b).

\section{EGR1 inhibition reduces the sepsis-induced inflammatory responses and improves the survival rate of endotoxemic mice}


Given the important role of EGR1 in the upregulation of ADAM17 expression in LPS-induced cells, we investigated whether EGR1 silencing is involved in the attenuation of the inflammatory response in vivo. ADAM17 gene expression was decreased in the lung and liver tissues of As-ODN-pretreated endotoxemia mice compared with those of LPS-treated control mice, and this effect was accompanied by the downregulation of endothelial cell injury factors, including ICAM1 and VCAM1 (Fig. 5b, c). In addition, As-ODN administration afforded mice a significant survival benefit following sepsis for 72 hours (Fig. $5 \mathrm{~d}$ ). Plasma levels of IL-1 13 (68.4 \pm 16.3 $\mathrm{pg} / \mathrm{mL}, P=0.026), \mathrm{IL}-6(761.5 \pm 775.0 \mathrm{pg} / \mathrm{ml}, P=0.015)$, and TNF-a $(199.5 \pm 159.7 \mathrm{pg} / \mathrm{ml}, P=0.0411)$ in As-ODN-pretreated endotoxemia mice were significantly decreased compared with those in the LPS-treated control mice $(102.50 \pm 28.68 \mathrm{pg} / \mathrm{ml}, 2914.0 \pm$ $1540.0 \mathrm{pg} / \mathrm{ml}, 458.8 \pm 147.5 \mathrm{pg} / \mathrm{ml}$, respectively) at 6 hours post-LPS injection (Fig. 5e).

\section{Discussion}

Convincing evidence demonstrates that ADAM17 SNPs play a crucial role in driving the inflammatory response during the course of inflammation-related diseases, which led us to focus on the relationship between genetic variations in ADAM17 and sepsis. Our previous study demonstrated the clinical relevance of five SNPs in the promoter region of ADAM17 in the susceptibility and progression of sepsis by showing that rs 12692386 is a susceptibility locus for the development of sepsis and is potentially involved in the regulation of ADAM17 transcriptional expression [14]. In this study, a larger cohort from multiple centers was used to validate the association between ADAM17 polymorphisms and sepsis in the Chinese Han population. Our analysis demonstrated that patients with the ADAM17 -172AG/GG genotype exhibit a trend toward aggravated sepsis development from sepsis subtype to severe sepsis and septic shock. Additionally, based on a Kaplan-Meier survival analysis, the 28-day survival in septic patients with $-172 \mathrm{GA} / \mathrm{GG}$ genotypes was much worse than in the AA genotype carriers, which is consistent with our previous results. We further explored the mechanisms underlying the SNP-mediated modulation of ADAM17 expression and the inflammatory response, which ultimately contributed to the development of sepsis.

ADAM17, which is also recognized as tumor necrosis factor alpha-converting enzyme (TACE), mediates inflammatory responses through the ectodomain shedding of pro-inflammatory cytokines in the transmembrane[32, 33]. The genomic deletion or pharmacologic inhibitor of ADAM17 in neutrophils and leukocytes results in evident resistance against LPS-induced endotoxemia and rescues mice from lethal septic shock $[15,16]$. It is undisputed that ADAM17 plays an important role in the process of sepsis. Our previous study indicated that ADAM17 expression is significantly increased in severe septic patients compared with mild sepsis cases [13]. Our results obtained in this study showed that the expression of ADAM17 was significantly higher in the MODS subgroup compared with the non-MODS subgroup. ADAM17 expression was significantly decreased in the sepsis patients who survived during the 28-day period compared with the non-surviving patients. These results indicated that ADAM17 might exert a significant effect on the progression of sepsis and might also serve as an indicator of disease severity. Many studies on inflammatory diseases have demonstrated that ADAM17 polymorphisms influence the expression of ADAM17 mRNA via potential allele-specific transcription factor-binding sites. A recent study revealed that the ADAM17 m1254 A>G polymorphism is located at a binding site for the transcription factor HNF1A/B [34]. Another study suggested that the ADAM17 i33708 A $>$ G polymorphism is located at a binding site for hepatocyte nuclear factor-2 (FOXA2), and available evidence indicates a regulatory role for the ADAM17 i62781 G>T polymorphism in the expression of the ADAM17 gene $[35,36]$. Moreover, it has been reported that the ADAM17 -172GA/GG genotype is related to higher ADAM17 expression, which results in increased susceptibility to human abdominal aortic aneurysm [37]. In the present study, LPS-stimulated PBMCs isolated from healthy individuals who carry the ADAM17 AG/GG genotypes displayed a significantly higher expression of ADAM17 and inflammatory factors compared with control PBMCs. Based on the results, we inferred that the ADAM17 SNP upregulates ADAM17 expression and the inflammatory response via the specific binding of transcriptional enhancers or other regulatory elements to the promoter region of ADAM17, which ultimately results in sepsis progression.

EGR1, a member of the early gene family, is an 80-kD transcription factor that preferentially binds to the GC-rich sequence 5'GCGGGGGCG-3' in DNA in a zinc-dependent manner [38, 39]. In inflammation-related diseases, EGR1 has been implicated in the transcription of various genes, including TNF-a, M-CSF, TF, ICAM1, CD44, EGF-R, and TGF- $\beta$ via its interaction with GC-rich regions, which play pivotal roles in various inflammatory-related diseases [40-49]. The ADAM17 rs12692386 is located within the gene promoter region and has binding sites for transcriptional regulatory elements. A bioinformatics analysis showed a conserved putative transcription factor-binding site for EGR1 in the 5'-UTR of human ADAM17. Our results showed that the presence of EGR1 significantly upregulated the expression of ADAM17 and substrates in LPS-stimulated cells, whereas these effects were completely abrogated by EGR1 knockdown. Thus, we speculated that EGR1 is an upstream regulator of the ADAM17 gene via allele-specific binding to the promoter region, and this binding is affected by the ADAM17 SNP. The ChIP-seq analysis results validated the binding 
of EGR1 to the ADAM17 promoter, and luciferase reporter assays further confirmed that ADAM17 A>G at the -172 position upregulated the affinity of EGR1 to the ADAM17 promoter region. These results provide strong evidence showing that the $G$ allele at the -172 position of the ADAM17 promoter has higher binding affinity for EGR1 than the A allele and thus appears to play a pivotal role in enhancing ADAM17 promoter activity and upregulating ADAM17 transcription.

In this case-control study, we confirmed that the ADAM17 -172A>G polymorphism is associated with susceptibility to and progression of sepsis, and in vitro experiments of the molecular mechanism showed that this variant of ADAM17 can influence sepsis progression by altering the binding affinity of EGR1 to the ADAM17 promoter region. Based on our results, carriers of the ADAM17 -172AG/GG genotype exhibit a worse prognosis. Moreover, the in vitro experiments revealed that EGR1 silencing resulted in decreased expression of cytokines and a lower cell apoptosis rate compared with the LPS group. Additionally, we found that the inhibition of EGR1 can reduce the inflammatory response and improve the survival of LPS-induced endotoxemia mice. Our study provides evidence indicating that the ADAM17 polymorphism is significantly associated with the progression of sepsis, and this association might aid the early identification and intervention of patients at risk for developing severe sepsis or septic shock. In addition, our exploration of the mechanism of susceptibility loci identified EGR1 as an important therapeutic target of sepsis.

Several limitations of this study should be mentioned. First, although inclusion and exclusion criteria were established to achieve greater sample homogeneity, we cannot preclude the possibility of unknown potential confounders, such as the pre-existing conditions of the patients with sepsis, which might affect the genetic results and observations in this case-control study. Second, all the subjects enrolled in this study belonged to the Chinese Han population, and a larger sample and multiethnic population are required to analyze the heterogeneity of the research results. In addition, though this study focused on the correlation between ADAM17 gene polymorphism and the progress of sepsis rather than the occurrence, the statistically significant differences in age and sex ratio were existed between the control group and the sepsis group, may also resulting in the imbalance of clinical baseline between the two groups, that was hard to excluded. Also, in animal experiments, the sampling time is single, and gene knockout mice are preferred to verify the hypothesis of this study.

\section{Conclusions}

In this study, we confirm that the ADAM17 -172A>G polymorphism confers susceptibility to the development of sepsis and poor prognosis. Carriers of the $\mathrm{G}$ allele exhibit higher expression levels of ADAM17 mRNA and inflammatory factors. In addition, EGR1 acts as a transcription factor targeting the $5^{\prime}-$ UTR of the ADAM17 gene, and the $-172 A>G$ variation upregulates ADAM17 expression by altering this binding site and thereby leads to progression of the inflammatory response and sepsis (Fig. 6).

\section{List Of Abbreviations}

ADAM17: A disintegrin and metalloproteinase 17; EGR1: Early growth response 1; SNP: Single-nucleotide polymorphism; ChIP-qPCR: Chromatin immunoprecipitation-quantitative Polymerase Chain Reaction; RIPA: Radio-Immunoprecipitation Assay; ELISA: Enzymelinked immunosorbent assay; TNF-a: Tumor necrosis factor alpha; IL-1ß: Interleukin-1 beta; IL-6: Interleukin-6; VCAM-1: vascular cell adhesion molecule-1; ICAM1: intercellular cell adhesion molecule-1; APACHEIl: acute physiology and chronic health evaluation II; cDNA: complementary deoxyribonucleic acid; PBMC: peripheral blood mononuclear cell; THP-1: Human acute monocytic leukemia cell line; RAW264.7 cell: mouse leukemia cells of monocyte macrophage; HUVECs: human umbilical vascular endothelial cells; HEK293T: human embryonic kidney cells; Ad-EGR1: adenoviruses containing EGR1; As-ODN: EGR1 antisense oligonucleotide; S-ODN: EGR1 sense ODN; LPS: Lipopolysaccharides; SP1: specificity protein 1; AP2a :TFAP2A; EGF-R: endothelial growth factor-receptor; TGF- $\beta$ : Transforming growth factor.

\section{Declarations}

\section{Ethics approval and consent to participate:}

All experiments on human subjects were conducted in accordance with the Declaration of Helsinki. This study was approved by the Ethical Committee of the Affiliated Hospital of Guangdong Medical University, the Second Affiliated Hospital of Guangdong Medical University, the Central Hospital of Wuhan and the Second Affiliated Hospital of Harbin Medical University, and written informed consent was obtained from all the healthy volunteers and patients. 
Consent for publication:

Not applicable

Availability of data and materials:

All data generated or analysed during this study are included in this published article.

\section{Competing interests:}

The authors declare that they have no competing interests.

\section{Funding:}

This work was supported by the Natural Science Foundation of Guangdong Province (2019A1515010933; 2020A1515010109), the Novel Coronavirus Infection Prevention technology and product project of Zhanjiang City (2020E01007), the National Nature Science Foundation of China (81772048 and 81671181) and the Fund of Southern Marine Science and Engineering Guangdong Laboratory (Zhanjiang)(ZJW-2019-007).

\section{Authors' contributions}

YS, JH and LC conceived and designed the experiments, and participated in its design and coordination and helped to draft the manuscript and revise the manuscript. YS, WZ and TZ collected the samples and clinical data, performed the experiments and the statistical analysis and drafted the manuscript. FL, YH, LL, YC, NW, HC and FC collected the samples and clinical data and helped to perform the experiments. All authors read and approved the final manuscript.

\section{Acknowledgements:}

Not applicable

\section{References}

1. Hotchkiss RS, Moldawer LL, Opal SM, et al. Sepsis and septic shock. Nat Rev Dis Primers. 2016;2:16045.

2. Prescott HC, Angus DC. Enhancing Recovery From Sepsis: A Review. JAMA. 2018;319(1):62-75.

3. Venkatesh B, Finfer S, Cohen J, et al. Adjunctive Glucocorticoid Therapy in Patients with Septic Shock. N Engl J Med. 2018;378(9):797-808

4. Angus DC, van der Poll T. Severe sepsis and septic shock. N Engl J Med. 2013;369(9):840-851.

5. Black RA, Rauch CT, Kozlosky CJ, et al. A metalloproteinase disintegrin that releases tumour-necrosis factor-alpha from cells. Nature. 1997;385(6618):729-733.

6. Rose-John S. ADAM17, shedding, TACE as therapeutic targets. Pharmacol Res. 2013;71:19-22.

7. Yan I, Schwarz J, Lucke K, et al. ADAM17 controls IL-6 signaling by cleavage of the murine IL-6Ralpha from the cell surface of leukocytes during inflammatory responses. J Leukoc Biol. 2016;99(5):749-760.

8. Garton KJ, Gough PJ, Philalay J, et al. Stimulated shedding of vascular cell adhesion molecule 1 (VCAM-1) is mediated by tumor necrosis factor-alpha-converting enzyme (ADAM 17). J Biol Chem. 2003;278(39):37459-37464.

9. Scheller J, Chalaris A, Garbers C, et al. ADAM17: a molecular switch to control inflammation and tissue regeneration. Trends Immunol. 2011;32(8):380-387.

10. Mishra HK, Ma J, Walcheck B. Ectodomain Shedding by ADAM17: Its Role in Neutrophil Recruitment and the Impairment of This Process during Sepsis. Front Cell Infect Microbiol. 2017;7:138.

11. Dreymueller D, Martin C, Kogel T, et al. Lung endothelial ADAM17 regulates the acute inflammatory response to lipopolysaccharide. EMBO Mol Med. 2012;4(5):412-423.

12. Kermarrec N, Selloum S, Plantefeve G, et al. Regulation of peritoneal and systemic neutrophil-derived tumor necrosis factor-alpha release in patients with severe peritonitis: role of tumor necrosis factor-alpha converting enzyme cleavage. Crit Care Med. 2005;33(6):1359-1364. 
13. Shao Y, He J, Chen F, et al. Association Study Between Promoter Polymorphisms of ADAM17 and Progression of Sepsis. Cell Physiol Biochem. 2016;39(4):1247-1261.

14. Mohler KM, Sleath PR, Fitzner JN, et al. Protection against a lethal dose of endotoxin by an inhibitor of tumour necrosis factor processing. Nature. 1994;370(6486):218-220.

15. Horiuchi K, Kimura T, Miyamoto T, et al. Cutting edge: TNF-alpha-converting enzyme (TACE/ADAM17) inactivation in mouse myeloid cells prevents lethality from endotoxin shock. J Immunol. 2007;179(5):2686-2689.

16. Mishra HK, Johnson TJ, Seelig DM, et al. Targeting ADAM17 in leukocytes increases neutrophil recruitment and reduces bacterial spread during polymicrobial sepsis. J Leukoc Biol. 2016;100(5):999-1004.

17. Li Y, Cui LL, Li QQ, et al. Association between ADAM17 promoter polymorphisms and ischemic stroke in a Chinese population. Journal of atherosclerosis and thrombosis. 2014;21(8):878-893.

18. Peng Q, Deng Y, Yang X, et al. Genetic variants of ADAM17 are implicated in the pathological process of Kawasaki disease and secondary coronary artery lesions via the TGF-beta/SMAD3 signaling pathway. Eur J Pediatr. 2016;175(5):705-713.

19. Morange PE, Tregouet DA, Godefroy T, et al. Polymorphisms of the tumor necrosis factor-alpha (TNF) and the TNF-alpha converting enzyme (TACE/ADAM17) genes in relation to cardiovascular mortality: the AtheroGene study. J Mol Med (Berl). 2008; 86(10):1153-1161.

20. Sukhatme VP. Early transcriptional events in cell growth: the Egr family. J Am Soc Nephrol. 1990;1(6):859-866.

21. Gashler A, Sukhatme VP. Early growth response protein 1 (Egr-1): prototype of a zinc-finger family of transcription factors. Prog Nucleic Acid Res Mol Biol. 1995;50:191-224.

22. Shin SY, Kim JH, Baker A, et al. Transcription factor Egr-1 is essential for maximal matrix metalloproteinase-9 transcription by tumor necrosis factor alpha. Mol Cancer Res. 2010;8(4):507-519.

23. Nishi H, Nishi KH, Johnson AC. Early Growth Response-1 gene mediates up-regulation of epidermal growth factor receptor expression during hypoxia. Cancer Res. 2002;62(3):827-834.

24. Khachigian LM, Lindner V, Williams AJ, Collins T. Egr-1-induced endothelial gene expression: a common theme in vascular injury. Science. 1996;271(5254):1427-1431.

25. Shi L, Kishore R, McMullen MR, Nagy LE. Lipopolysaccharide stimulation of ERK1/2 increases TNF-alpha production via Egr-1. Am J Physiol Cell Physiol. 2002;282(6):C1205-1211.

26. Pawlinski R, Pedersen B, Kehrle B, et al. Regulation of tissue factor and inflammatory mediators by Egr-1 in a mouse endotoxemia model. Blood. 2003;101(10):3940-3947.

27. Coleman DL, Bartiss AH, Sukhatme VP, et al. Lipopolysaccharide induces Egr-1 mRNA and protein in murine peritoneal macrophages. J Immunol. 1992;149(9):3045-3051.

28. Shan J, Dudenhausen E, Kilberg MS. Induction of early growth response gene 1 (EGR1) by endoplasmic reticulum stress is mediated by the extracellular regulated kinase (ERK) arm of the MAPK pathways. Biochim Biophys Acta Mol Cell Res. 2019;1866(3):371-381.

29. Ngiam N, Post M, Kavanagh BP: Early growth response factor-1 in acute lung injury. Am J Physiol Lung Cell Mol Physiol. 2007;293(5):L1089-1091.

30. Chen F, Wang Y, Zhang W, et al. A Functional Polymorphism-Mediated Disruption of EGR1/ADAM10 Pathway Confers the Risk of Sepsis Progression. mBio. 2019;10(4):e01663-19.

31. Zhang Y, Shi G, Zheng J, et al. The protective effect of Egr-1 antisense oligodeoxyribonucleotide on myocardial injury induced by ischemia-reperfusion and hypoxia-reoxygenation. Cell Physiol Biochem. 2008;22(5-6):645-652.

32. Lambrecht BN, Vanderkerken M, Hammad H. The emerging role of ADAM metalloproteinases in immunity. Nat Rev Immunol. 2018;8(12):745-758.

33. Grotzinger J, Lorenzen I, Dusterhoft S. Molecular insights into the multilayered regulation of ADAM17: The role of the extracellular region. Biochim Biophys Acta Mol Cell Res. 2017;1864(11 Pt B):2088-2095.

34. Stride A, Ellard S, Clark P, et al. Beta-cell dysfunction, insulin sensitivity, and glycosuria precede diabetes in hepatocyte nuclear factor-1alpha mutation carriers. Diabetes care. 2005;28(7):1751-1756.

35. Wolfrum C, Shih DQ, Kuwajima S, Norris AW, Kahn CR, Stoffel M. Role of Foxa-2 in adipocyte metabolism and differentiation. J Clin Invest. 2003;112(3):345-356. 
36. Dixon AL, Liang L, Moffatt MF, et al. A genome-wide association study of global gene expression. Nat Genet. 2007;39(10):12021207.

37. Li Y, Yang C, Ma G, et al. Analysis of ADAM17 polymorphisms and susceptibility to sporadic abdominal aortic aneurysm. Cell Physiol Biochem. 2014;33(5):1426-1438.

38. Wagner M, Schmelz K, Dorken B, Tamm I. Transcriptional regulation of human survivin by early growth response (Egr)-1 transcription factor. Int J Cancer. 2008;122(6):1278-1287.

39. Gashler AL, Swaminathan S, Sukhatme VP. A novel repression module, an extensive activation domain, and a bipartite nuclear localization signal defined in the immediate-early transcription factor Egr-1. Mol Cell Biol. 1993;13(8):4556-4571.

40. Katz S, Zsiros V, Doczi N, Kiss AL. Inflammation-Induced Epithelial-to-Mesenchymal Transition and GM-CSF Treatment Stimulate Mesenteric Mesothelial Cells to Transdifferentiate into Macrophages. Inflammation. 2018;41(5):1825-1834.

41. Krishnaraju K, Hoffman B, Liebermann DA. The zinc finger transcription factor Egr-1 activates macrophage differentiation in M1 myeloblastic leukemia cells. Blood. 1998;92(6):1957-1966.

42. Krishnaraju K, Nguyen HQ, Liebermann DA, Hoffman B. The zinc finger transcription factor Egr-1 potentiates macrophage differentiation of hematopoietic cells. Mol Cell Biol. 1995;15(10):5499-5507.

43. Cui MZ, Parry GC, Oeth P, et al. Transcriptional regulation of the tissue factor gene in human epithelial cells is mediated by Sp1 and EGR-1. J Biol Chem. 1996;271(5):2731-2739.

44. Bea F, Puolakkainen MH, McMillen T, et al. Chlamydia pneumoniae induces tissue factor expression in mouse macrophages via activation of Egr-1 and the MEK-ERK1/2 pathway. Circ Res. 2003;92(4):394-401.

45. Maltzman JS, Carmen JA, Monroe JG. Transcriptional regulation of the Icam-1 gene in antigen receptor- and phorbol esterstimulated B lymphocytes: role for transcription factor EGR1. J Exp Med. 1996;183(4):1747-1759.

46. Ghatak S, Markwald RR, Hascall VC, et al. Transforming growth factor beta1 (TGFbeta1) regulates CD44V6 expression and activity through extracellular signal-regulated kinase (ERK)-induced EGR1 in pulmonary fibrogenic fibroblasts. J Biol Chem. 2017; 292(25):10465-10489.

47. Alagappan D, Balan M, Jiang Y, et al. Egr-1 is a critical regulator of EGF-receptor-mediated expansion of subventricular zone neural stem cells and progenitors during recovery from hypoxia-hypoglycemia. ASN Neuro. 2013;5(3):183-193.

48. Baron V, Adamson ED, Calogero A, Ragona G, Mercola D. The transcription factor Egr1 is a direct regulator of multiple tumor suppressors including TGFbeta1, PTEN, p53, and fibronectin. Cancer Gene Ther. 2006;13(2):115-124.

49. Zhang M, Liao Y, Lonnerdal B. EGR-1 is an active transcription factor in TGF-beta2-mediated small intestinal cell differentiation. J Nutr Biochem. 2016;37:101-108.

\section{Tables}

Table 1: Clinical characteristics of sepsis patients and healthy controls. 


\begin{tabular}{|c|c|c|c|c|}
\hline Variable & $\begin{array}{l}\text { Sepsis subtype } \\
\mathrm{n}=181\end{array}$ & $\begin{array}{l}\text { Severe sepsis } \\
\mathrm{n}=368\end{array}$ & $\begin{array}{l}\text { Septic shock } \\
n=354\end{array}$ & $\begin{array}{l}\text { Healthy controls } \\
n=1150\end{array}$ \\
\hline \multicolumn{5}{|l|}{ Demographics } \\
\hline Age, years, mean \pm SD & $60.1 \pm 15.0$ & $61.0 \pm 18.5$ & $59.9 \pm 19.1$ & $48.1 \pm 15.2$ \\
\hline Male/female, number & $102 / 79$ & $281 / 87$ & $234 / 120$ & $712 / 438$ \\
\hline \multicolumn{5}{|l|}{ Source of infection, no./total no. (\%) } \\
\hline Respiratory tract infection & $83 / 181(45.9)$ & $286 / 368(77.7)$ & $143 / 354(40.4)$ & N.A \\
\hline Abdominal infection & 29/181(16.0) & $7 / 368(1.9)$ & $76 / 354(21.4)$ & N.A \\
\hline Urinary tract infection & $9 / 181(5.0)$ & $17 / 368(4.6)$ & $38 / 354(10.7)$ & N.A \\
\hline Catheter-associated infection & $0 / 181(0.0)$ & $11 / 36(3.0)$ & $7 / 354(2.0)$ & N.A \\
\hline Brain & $11 / 181(6.1)$ & $2 / 368(0.5)$ & $8 / 354(2.3)$ & N.A \\
\hline Others & $3 / 181(1.6)$ & $10 / 368(2.8)$ & $30 / 354(8.5)$ & N.A \\
\hline \multicolumn{5}{|l|}{ Identified pathogen, no./total no. (\%) } \\
\hline Gram-positive(G+) & 20/181(11.1) & $56 / 368(15.2)$ & $33 / 354(9.3)$ & N.A \\
\hline Gram-negative(G-) & 67/181(37.0) & $102 / 368(27.7)$ & $127 / 354(35.9)$ & N.A \\
\hline Mixed Gram-negative and -positive & 27/181(14.9) & $47 / 368(12.8)$ & 28/354(7.9) & N.A \\
\hline Fungus & $35 / 181(19.4)$ & $18 / 368(4.9)$ & $60 / 354(17.0)$ & N.A \\
\hline Polymicrobial & 18/181(9.9) & $68 / 368(18.5)$ & $23 / 354(6.5)$ & N.A \\
\hline Negative blood culture & 158/181(87.3) & $168 / 368(45.7)$ & $209 / 354(59.0)$ & N.A \\
\hline \multicolumn{5}{|l|}{ Pathogenic bacteria, no./total no. (\%) } \\
\hline Acinetobacter baumannii & $58 / 181(32.0)$ & $70 / 368(19.0)$ & $109 / 354(30.8)$ & N.A \\
\hline Moniliaalbican & $32 / 181(17.7)$ & $16 / 368(4.3)$ & $37 / 354(10.5)$ & N.A \\
\hline Yeast sample sporphyte & $8 / 181(4.4)$ & $35 / 368(9.5)$ & $16 / 354(4.5)$ & N.A \\
\hline Aspergillus & $2 / 181(1.1)$ & $19 / 368(5.2)$ & $17 / 354(4.8)$ & N.A \\
\hline Klebsiella pneumoniae & 13/181(7.2) & $19 / 368(5.2)$ & $29 / 354(8.2)$ & N.A \\
\hline Pseudomonas aeruginosa & 26/181(14.4) & $57 / 368(15.5)$ & $26 / 354(7.3)$ & N.A \\
\hline Staphylococcus aureus & 13/181(17.1) & $31 / 368(3.5)$ & $35 / 354(23.2)$ & N.A \\
\hline Escherichia coli & $4 / 181(2.2)$ & $67 / 368(18.2)$ & $26 / 354(7.3)$ & N.A \\
\hline Others & 7/181(3.9) & $72 / 368(19.6)$ & $12 / 354(3.4)$ & N.A \\
\hline APACHE II score, mean \pm SD & $19.1 \pm 6.5$ & $19.8 \pm 6.2$ & $21.0 \pm 7.1$ & N.A \\
\hline
\end{tabular}

N.A: not applicable; APACHE II: Acute Physiology and Chronic Health Evaluation Il; Continuous data are expressed as the mean \pm SD.

Table 2: Genotype and allele frequencies distribution of the ADAM17 rs12692386 in the different sepsis status. 


\begin{tabular}{|c|c|c|c|c|c|c|c|}
\hline rs12692386 & $\begin{array}{l}\text { Sepsis } \\
\text { subtype } \\
n=181(\%)\end{array}$ & $\begin{array}{l}\text { Severe sepsis } \\
n=368(\%)\end{array}$ & $\begin{array}{l}\text { Septic shock } \\
n=354(\%)\end{array}$ & $\begin{array}{l}\text { Severe sepsis \& } \\
\text { septic shock } \\
n=722(\%)\end{array}$ & $\mathrm{P} 1$ (P1*) & P2 (P2*) & P3 (P3*) \\
\hline AA & 128/181(70.7) & $199 / 368(54.1)$ & $204 / 354(58.6)$ & 403/722(55.8) & $\begin{array}{l}0.0002 \\
(0.0005)\end{array}$ & $\begin{array}{l}0.0032 \\
(0.0032)\end{array}$ & $\begin{array}{l}0.0003 \\
(0.0005)\end{array}$ \\
\hline $\mathrm{GA} \& \mathrm{GG}$ & $53 / 181(29.3)$ & $169 / 368(45.9)$ & $150 / 354(42.4)$ & $319 / 722(42.2)$ & - & - & - \\
\hline A & $300 / 362(82.9)$ & $546 / 736(74.2)$ & $543 / 708(76.7)$ & $1089 / 1444(75.4)$ & $0.0013(0.0039)$ & $\begin{array}{l}0.0193 \\
(0.0193)\end{array}$ & $\begin{array}{l}0.0026 \\
(0.0039)\end{array}$ \\
\hline G & $62 / 362(17.1)$ & 190/736(25.8) & 165/708(23.3) & $355 / 1444(24.6)$ & - & - & - \\
\hline
\end{tabular}

P1: sepsis subtype versus severe sepsis; P2: sepsis subtype versus septic shock; P3, sepsis subtype versus severe sepsis \& septic shock. *False discovery rate-adjusted $P$-value for multiple hypotheses testing using the Benjamin-Hochberg method.

Table 3: Genotype and allele frequencies distribution of the ADAM17 rs12692386 in the cases and controls.

\begin{tabular}{|llllll|}
\hline Genotypes & $\begin{array}{l}\text { Sepsis } \\
n=903(\%)\end{array}$ & $\begin{array}{l}\text { Control } \\
n=1150(\%)\end{array}$ & P & P* & OR (95\%Cl) \\
\hline AA & $531 / 903(58.8)$ & $697 / 1150(60.6)$ & 0.3043 & 0.5436 & - \\
\hline AG & $327 / 903(36.2)$ & $384 / 1150(33.4)$ & - & - & - \\
\hline GG & $45 / 903(5.0)$ & $69 / 1150(6.0)$ & - & - & - \\
\hline AA\&AG & $858 / 903(95.0)$ & $1081 / 1150(94.0)$ & 0.3181 & 0.5436 & $1.217(0.827,1.791)$ \\
\hline AG\&GG & $372 / 903(41.9)$ & $453 / 1150(39.4)$ & 0.4077 & 0.5436 & $1.078(0.902,1.287)$ \\
\hline A & $1389 / 1806(76.9)$ & $1778 / 2300(77.3)$ & - & - & - \\
\hline G & $417 / 1806(23.1)$ & $522 / 2300(22.7)$ & 0.7654 & 0.7654 & $0.978(0.845,1.132)$ \\
\hline
\end{tabular}

OR: odds ratio; $95 \% \mathrm{Cl}$ : 95\% confidence interval. *False discovery rate-adjusted P-value for multiple hypotheses testing using the Benjamin-Hochberg method.

Table 4: Genotype and allele frequencies distribution between 28-day surviving and non-surviving sepsis patients.

\begin{tabular}{|llllll|}
\hline rs12692386 & $\begin{array}{l}\text { Survivors } \\
\mathrm{n}=751(\%)\end{array}$ & $\begin{array}{l}\text { Non-survivors } \\
\mathrm{n}=152(\%)\end{array}$ & $\mathbf{P}$ & $\mathrm{P} *$ & OR (95\%Cl) \\
\hline AA & $456 / 751(60.7)$ & $75 / 152(49.3)$ & 0.0178 & 0.0237 & - \\
\hline AG & $262 / 751(34.9)$ & $65 / 152(42.8)$ & - & - & - \\
\hline GG & $33 / 751(4.4)$ & $12 / 152(7.9)$ & - & - & - \\
\hline AA\&AG & $718 / 751(95.6)$ & $140 / 152(92.1)$ & 0.0705 & 0.0705 & $1.865(0.940,3.700)$ \\
\hline AG\&GG & $295 / 751(39.3)$ & $77 / 152(50.7)$ & 0.0094 & 0.0188 & $0.630(0.444,0.894)$ \\
\hline A & $1174 / 1502(78.2)$ & $215 / 304(70.7)$ & - & - & - \\
\hline G & $328 / 1502(21.8)$ & $89 / 304(29.3)$ & 0.0050 & 0.0188 & $1.482(1.125,1.952)$ \\
\hline
\end{tabular}

*False discovery rate-adjusted P-value for multiple hypotheses testing using the Benjamin-Hochbergmethod. 
Figures

a

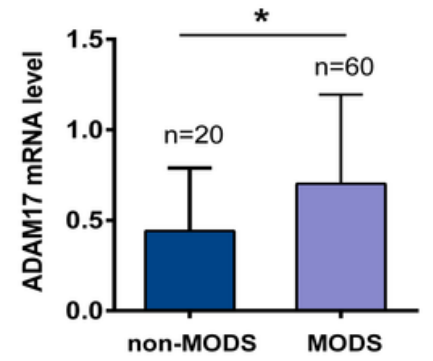

b

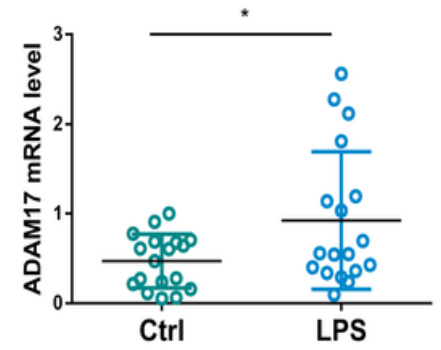

C

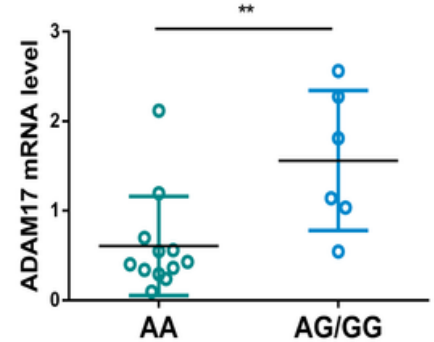

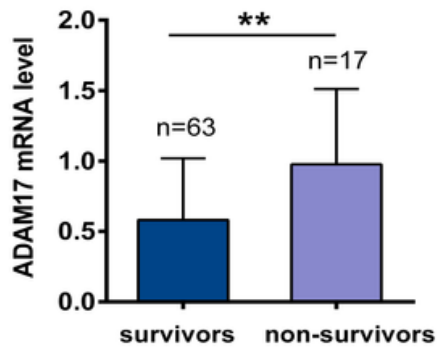
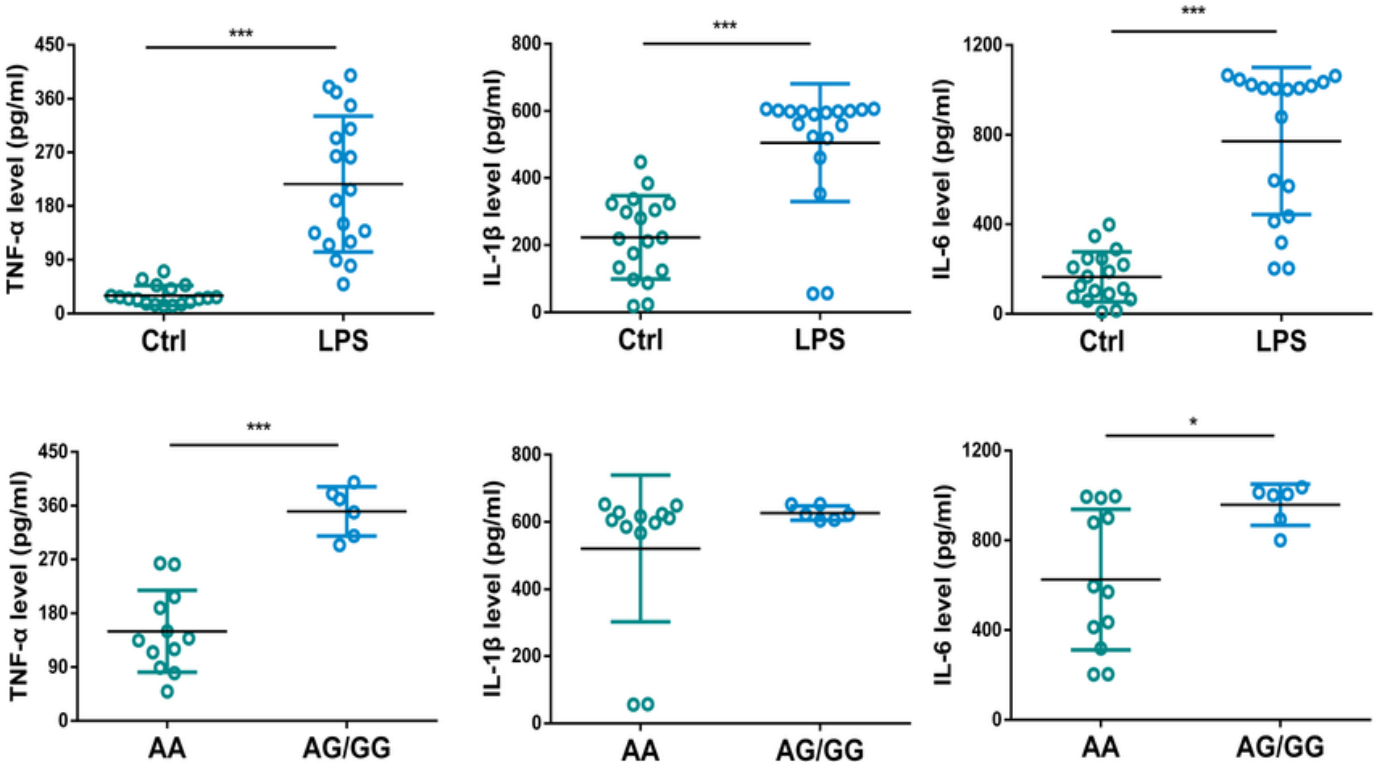

Figure 1

Impact of ADAM17 SNP on expression of ADAM17 and inflammatory factors. (a) The expression of ADAM17 in the 80 sepsis cases separated by the number of organ failure and patients' 28-day mortality. PBMCs of 36 healthy controls were isolated and randomly divided into LPS group ( $n=18$ ) (LPS: $800 \mathrm{ng} / \mathrm{mL}, 6 \mathrm{~h}$ ) and Control group ( $\mathrm{n}=18)$; (b) RT-qPCR analysis of ADAM17 expression in PBMCs and ELISA kit detection of cytokines (TNF-a, IL-1 $\beta$, and IL-6) in the culture medium of PBMCs; (c) The ADAM17 rs12692386 polymorphism of the above mentioned 18 subjects from the LPS group was genotyped and then divided into two groups: AA vs. $A G / G G$ ( $n=12$ for $A A$ carriers, and $n=6$ for $A G / G G$ carriers). The expressions of ADAM17 mRNA and inflammatory cytokines in these two groups were statistically analyzed. Error bar represent standard error of the mean (SD). ${ }^{\star} P<0.05,{ }^{\star} * P<0.01$, and ${ }^{* \star *} P<0.001$. 


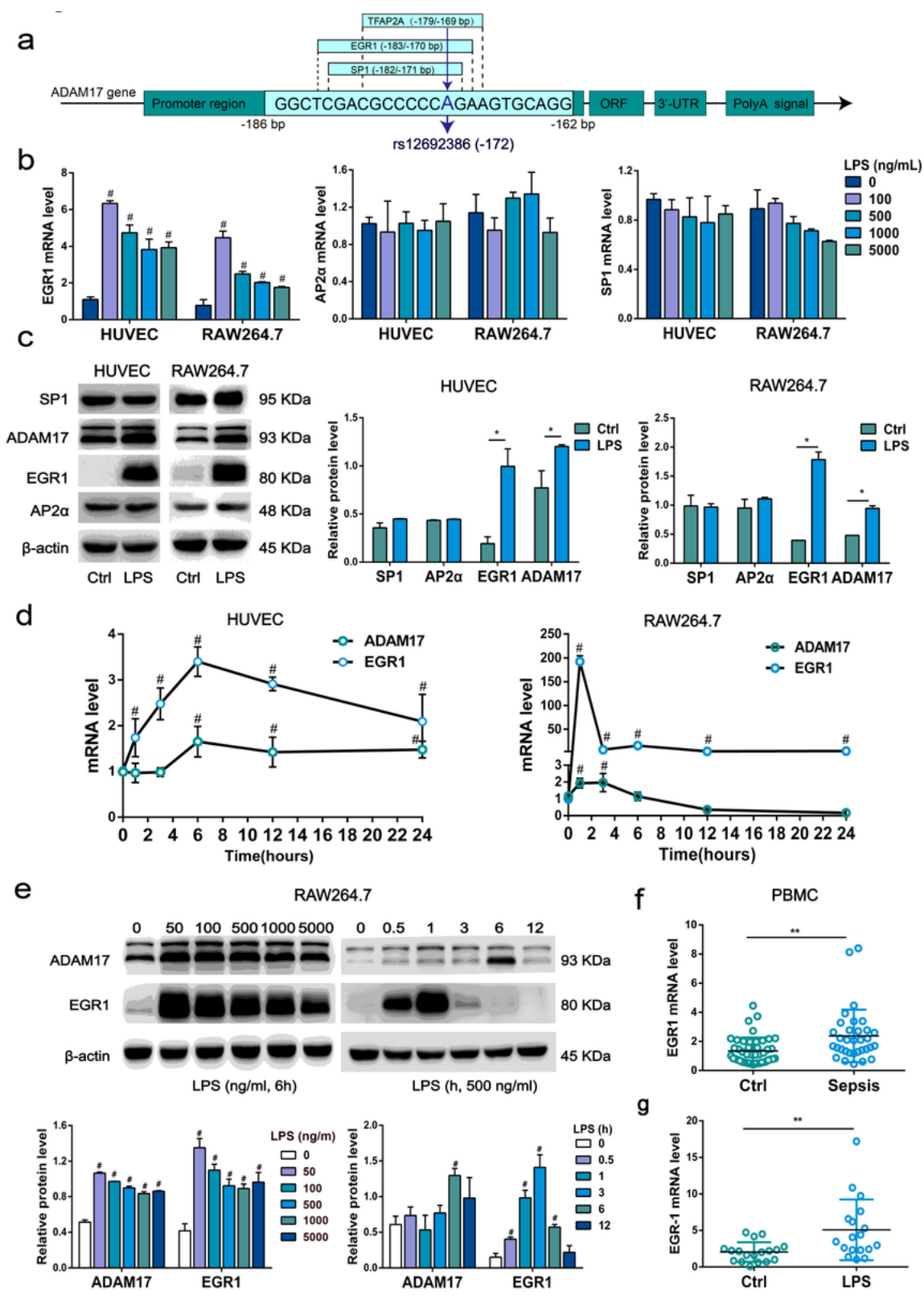

Figure 2

Expression of predicted transcription factors in sepsis. (a) Bioinformatics analysis of binding of transcription factors to the promoter region of ADAM17 around the -172 position; (b) RT-qPCR analysis of transcription factor (EGR1, AP2a, and SP1) expression in HUVECs and RAW264.7 cells with increasing doses of LPS $(0,100,500,1000,5000 \mathrm{ng} / \mathrm{mL})$ for 6 hours; (c) Western blot analysis of transcription factors and ADAM17 expressions in HUVECs and RAW264.7 treated with LPS (LPS: $1000 \mathrm{ng} / \mathrm{mL}$ for HUVECs and 500 $\mathrm{ng} / \mathrm{mL}$ for RAW264.7, both pretreated for 6 hours); (d) RT-qPCR analysis of EGR1 and ADAM17 expression in HUVECs and RAW264.7 cells with an increasing duration of LPS (LPS: $1000 \mathrm{ng} / \mathrm{mL}$ for HUVECs and $500 \mathrm{ng} / \mathrm{mL}$ for RAW264.7); (e) Western blot analysis of EGR1 and ADAM17 in RAW264.7 cells with increasing concentrations of LPS (6 hours) or various durations of LPS (500 ng/mL); (f) RT-qPCR analysis of EGR1 expression in PBMCs isolated from 33 sepsis patients and 45 healthy controls; (g) RT-qPCR analysis of EGR1 expression in PBMCs isolated from 36 healthy controls. Error bar represent standard error of the mean (SD). \#P<0.05 vs. control group; ${ }^{*} \mathrm{P}<0.05$ and ${ }^{*} \mathrm{P}<0.01$. 
a

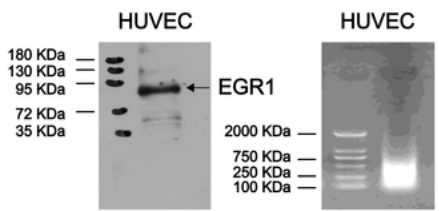

b

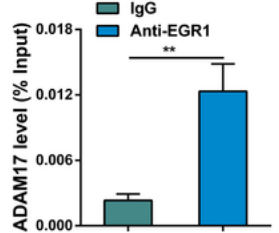

C

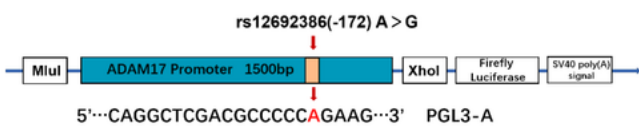

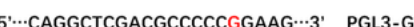
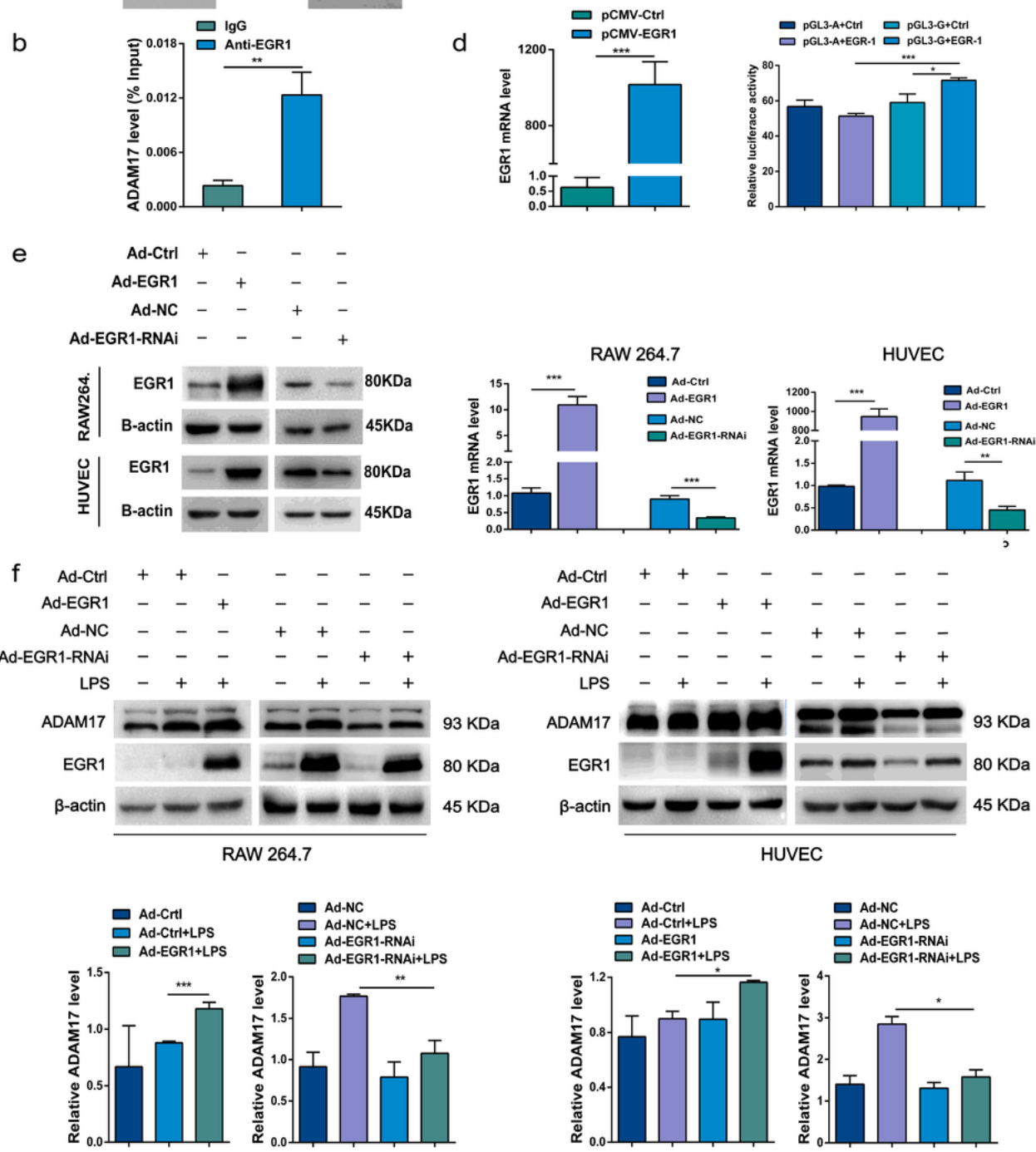

Figure 3

Binding of EGR1 to the ADAM17 promoter regulates the expression of ADAM17 in LPS-induced HUVECs and RAW264.7 cells. (a) Western blotting was used to determine the presence of EGR1 protein in HUVECs (left). Chromatin fragmentation was conducted to digest chromatins into 0.1-1 kb fragments, and the effective fragments were enriched within the size range 100-750 bp (right); (b) ChIP-qPCR was performed to identify the binding of EGR1 to the ADAM17 promoter region. Input samples were used as internal references; (c) ADAM17 plasmid constructs and functional promoter activities of rs12692386 in HEK293T cells. The promoter region of ADAM17 carrying the A or G alleles of rs 12692386 was cloned into pGL3 luciferase reporter vectors; (d) EGR1 overexpression in HEK293T cells was detected by RT-qPCR. The different ADAM17 plasmid constructs were cotransfected into cells with EGR1 for 48 hours, and the promoter activities were detected by dual-luciferase reporter assays; (e) EGR1 was overexpressed or silenced by adenovirus mediated gene modification in RAW264.7 cells and HUVECs; ( $f$ ) Western blotting was used to detect the impact of EGR1 on the expression of ADAM17 in sepsis. Error bar represent standard error of the mean (SD). ${ }^{*} P<0.05,{ }^{*} P<0.01$, and ${ }^{* \star *} P<0.001$. NC: negative control. 
a
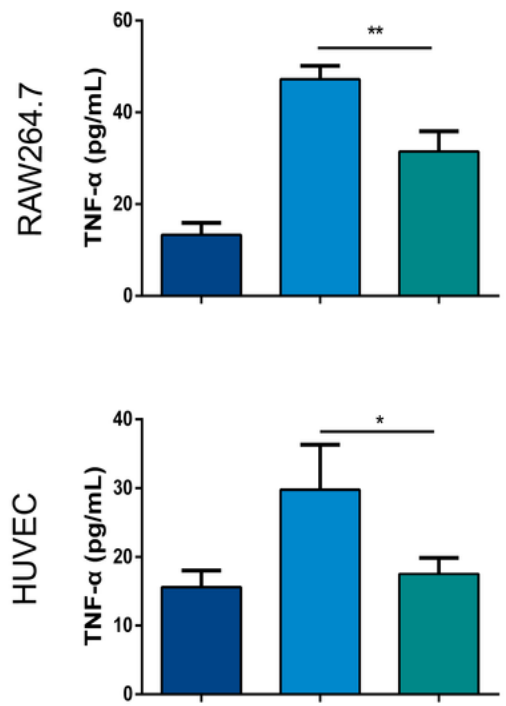

b

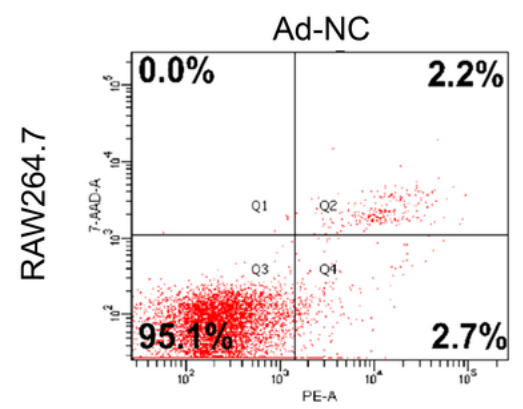

$\square$ Ad-NC

$\square$ Ad-NC+LPS

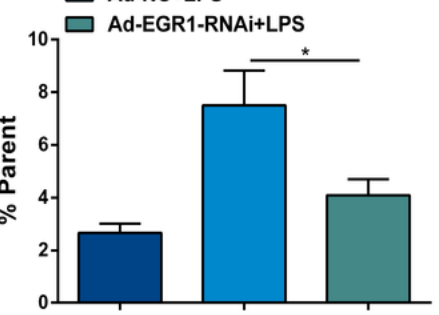

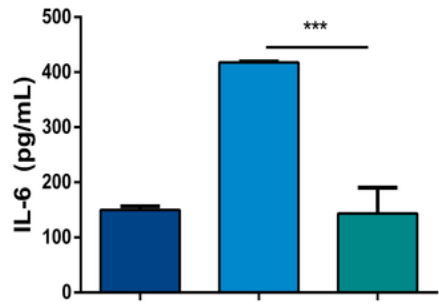

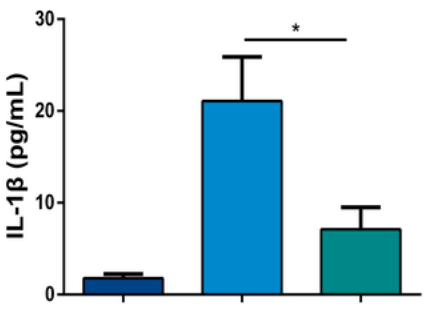

$\square$ Ad-NC

$\square$ Ad-NC+LPS
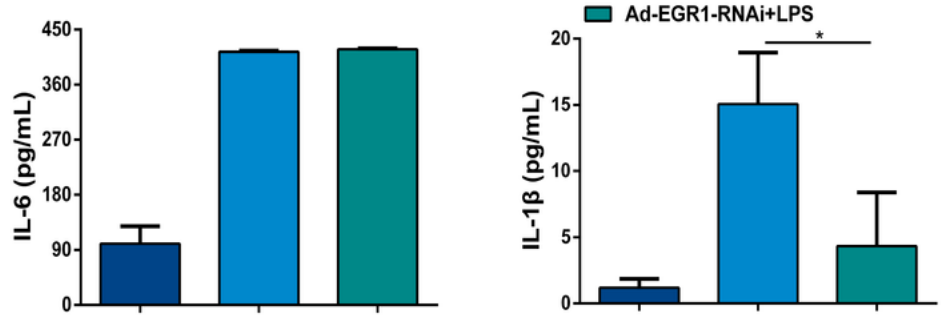

Ad-EGR1-RNAi+LPS
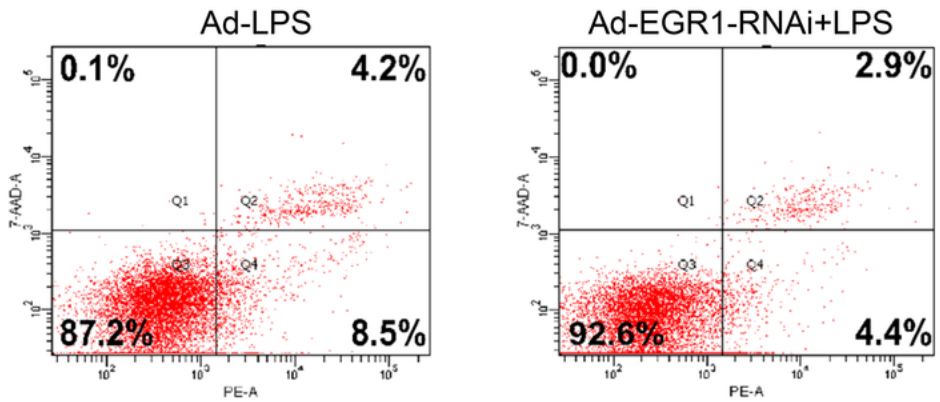

\section{Figure 4}

Inhibition of EGR1/ADAM17 pathway decreases inflammatory response. (a) Concentration of cytokines (TNF-a, IL-1- $\beta$ and IL-6) in cell culture medium was detected under conditions of EGR1 silencing in RAW264.7 cells after 6 hours of LPS stimulation; (b) Flow cytometry was performed to determine the effects of EGR1 silencing on apoptosis in LPS-stimulated RAW264.7 cells. Error bar represent standard error of the mean (SD). ${ }^{\star} \mathrm{P}<0.05,{ }^{\star \star} \mathrm{P}<0.01$, and ${ }^{* \star *} \mathrm{P}<0.001$. NC: negative control. 
a

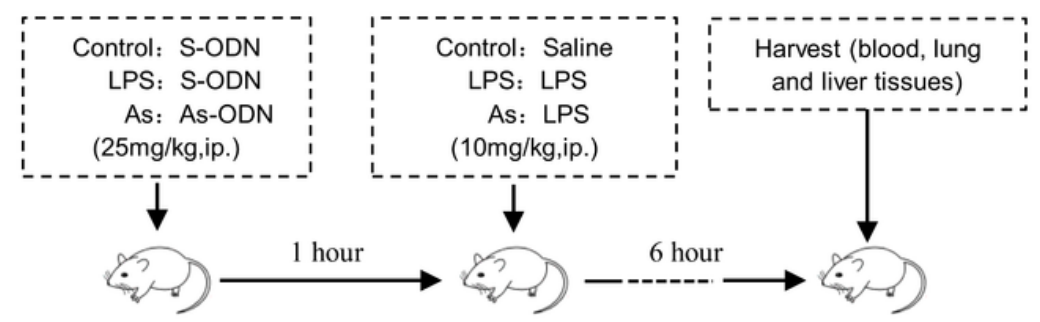

b
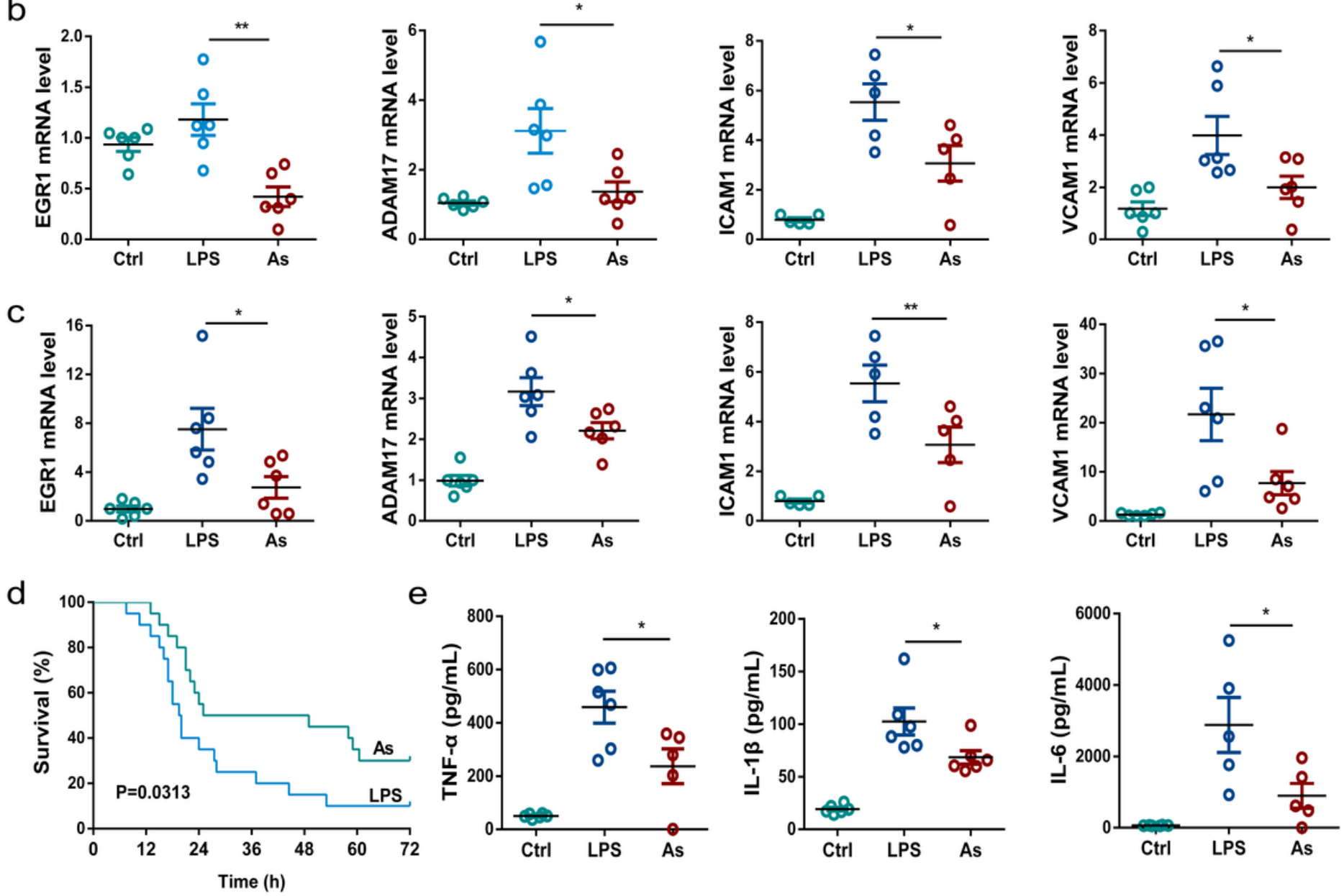

Figure 5

EGR1 silencing attenuates LPS-induced endotoxemia. (a) Schematic of experimental design and time line. Endotoxemia was induced in C57BL/ 6 mice by intraperitoneal injection of LPS $(10 \mathrm{mg} / \mathrm{kg})$, and control animals were administered equivalent volumes of normal saline. EGR1 As-ODN were delivered into mice by intraperitoneal injection 1 hour before LPS challenge; (b) Expressions of EGR1, ADAM17, ICAM1 and VCAM1 in lung tissues were detected by RT-qPCR (6 mice per group, LPS: 10 mg/kg); (c) Expressions of EGR1, ADAM17, ICAM1 and VCAM1 in liver tissues were detected by RT-qPCR; (d) Seventy-two-hour survival curves of endotoxemia mice given a lethal dose of LPS (20 mice per group, LPS: $20 \mathrm{mg} / \mathrm{kg}$ ). Data were analyzed by Log-rank Mantel-Cox survival test; (e) Expressionsof cytokines (IL-1- $\beta$, IL-6 and TNF- $a$ ) in the serum of mice challenged with LPS for 6 hours $(n=6)$. Error bar represent standard error of the mean (SD). ${ }^{\star} \mathrm{P}<0.05$ and ${ }^{*} \mathrm{P}<0.01$ vs. LPS group. ODN: oligonucleotide; As: antisense oligonucleotide (As-ODN). 


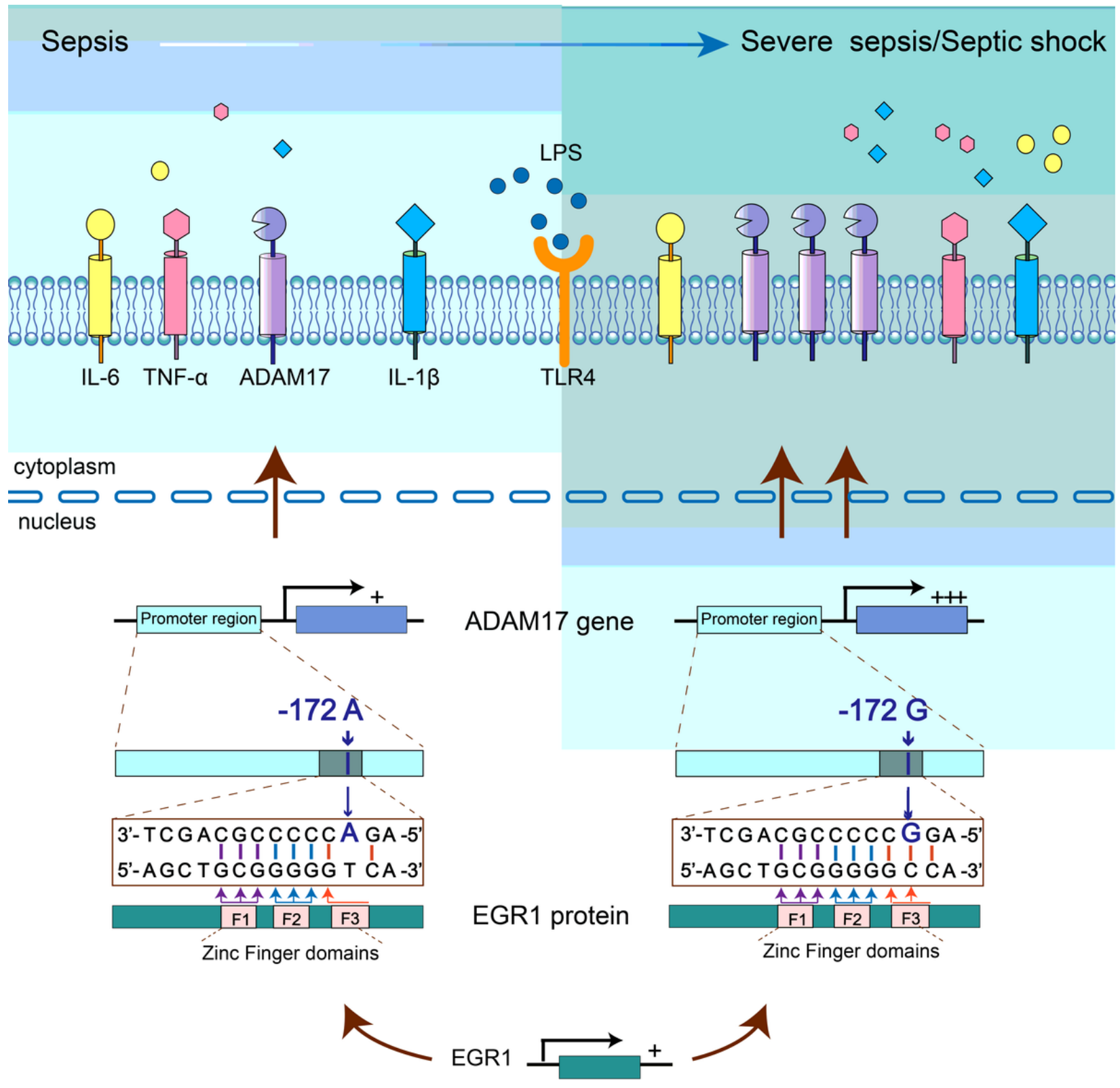

Figure 6

Cellular schematic of the molecular mechanism of ADAM17 SNP-mediated EGR1 modulation on ADAM17 expression in the progression of sepsis. The $A$ to $G$ mutation of $A D A M 17-172 A>G$ polymorphism increased the binding affinity of transcription factor EGR1 to ADAM17 promoter region, which transcriptionally elevated the expression of ADAM17 and ultimately exacerbated sepsis to severe sepsis/septic shock via increasing the cleavage activity of ADAM17 to substrates, including pro-inflammatory (IL-1 13 , IL-6, TNFa).

\section{Supplementary Files}

This is a list of supplementary files associated with this preprint. Click to download.

- Additionalfile5.pdf

- Additionalfile4.pdf

- Additionalfile3.pdf

- Additionalfile2.pdf

- Additionalfile1.pdf 